\title{
Statistical representation of clouds in a regional model and the impact on the diurnal cycle of convection during Tropical Convection, Cirrus and Nitrogen Oxides (TROCCINOX)
}

\author{
Jean-Pierre Chaboureau and Peter Bechtold \\ Laboratoire d'Aérologie, Observatoire Midi Pyrénées, Université Paul Sabatier and Centre National de la Recherche \\ Scientifique, Toulouse, France \\ Received 30 November 2004; revised 15 May 2005; accepted 14 June 2005; published 10 September 2005.
}

[1] A statistical cloud parameterization is presented, where in addition to earlier work the subgrid moisture/thermal variability is diagnostically determined as a sum of a turbulent and a convective contribution, the latter being a function of the convective mass flux. It is shown that the inclusion of a convective contribution allows us to reasonably reconstruct the time mean and variability of the variance of the conserved quantities total water and liquid water static energy. Application of the parameterization in a regional model during a 30-day period of the Tropical Convection, Cirrus and Nitrogen Oxides (TROCCINOX) experiment over southern Brazil demonstrates a clear improvement in the representation of the cloud fields. The subgrid cloud parameterization significantly reduces both the biases and phase between the observed and simulated brightness temperatures in the thermal infrared band and the observed and simulated precipitation intensities, resulting in an improved representation of the diurnal cycle of convection over land. The strong impact on the diurnal cycle is mainly a consequence of reduced surface fluxes due to cloud shading and a change in tropospheric stability due to additional condensation/sublimation.

Citation: Chaboureau, J.-P., and P. Bechtold (2005), Statistical representation of clouds in a regional model and the impact on the diurnal cycle of convection during Tropical Convection, Cirrus and Nitrogen Oxides (TROCCINOX), J. Geophys. Res., 110, D17103, doi:10.1029/2004JD005645.

\section{Introduction}

[2] Convection is an important generation mechanism not only for convective towers (covering typical area fractions of less than $10 \%$ ) but also of layered long-lived clouds through detrainment of cloud mass at tropospheric inversions (e.g., trade wind inversion, melting level, tropopause). In current mesoscale and global general circulation models deep convection is still generally represented by a mass flux convection parameterization [Arakawa and Schubert, 1974; Tiedtke, 1989]. Therefore Tiedtke [1993] and Teixeira [2001] suggested generation of convective clouds and their anvils through the detrainment rate of cloud water from the convection scheme as a source term in the prognostic equations for cloud condensate and cloud fraction.

[3] As an alternative to this more deterministic approach, a statistical description of clouds might be used. Since the early work of Mellor [1977], Sommeria and Deardorff [1977], and Bougeault [1981], numerous statistical formulations of clouds have been developed on the basis of either cloud-resolving model (CRM) data [Xu and Krueger, 1991; Cuijpers and Bechtold, 1995; Xu and Randall, 1996; Lenderink and Siebesma, 2000; Tompkins, 2002; Chaboureau

Copyright 2005 by the American Geophysical Union. 0148-0227/05/2004JD005645 and Bechtold, 2002], global data from a mesoscale model [Cusack et al., 1999], aircraft and balloon data [Wood and Field, 2000; Price and Wood, 2002], or using specified statistical distribution functions and trying to minimize some observational constraint like liquid water path or outgoing longwave radiation [Smith, 1990; Ricard and Royer, 1993; Bony and Emanuel, 2001]. The schemes mainly differ by the choice of the statistical quantity (either total water, saturation specific humidity, relative humidity or a combination of total water and liquid water temperature) used to derive cloud fraction and cloud condensate, the choice of the distribution function for this quantity (using either analytical or empirical functions), and the specification of the variance and/or higher-order moments of the statistical quantity.

[4] In a model study, Lohmann et al. [1999] compare different diagnostic statistical schemes and prognostic microphysical schemes and discuss how statistical schemes can be coupled to a prognostic microphysical scheme. One of the outcomes of their study was that the use of a prognostic microphysical scheme seems to be more appropriate for upper tropospheric long-lived cirrus clouds, whereas statistical cloud schemes seem to be superior for the representation of boundary layer clouds. In order to overcome the two main difficulties with the statistical approach that are (1) the coupling with prognostic equations for the cloud condensate and/or the cloud fraction and 
(2) the difficulty to represent the generation of clouds associated with deep convective events, Tompkins [2002] suggested to predict the variance of total water including several terms that are related to stratiform and convective contributions. His parameterization is also based on the CRM results of $X u$ and Krueger [1991] that showed a strong correlation between the convective mass flux and the cumulus cloud amount.

[5] Recently, using CRM data, Chaboureau and Bechtold [2002, hereinafter referred to as CB] showed that both boundary layer and deep convective clouds can be represented with the same statistical relations based on the subgrid variability (variances) of total water and liquid water static energy. They also presented a simple method to couple the statistical scheme with a prognostic formulation of the cloud condensate. However, during extensive tests of the scheme by colleagues at the European Centre for Medium Range Weather Forecast (ECMWF) using data from the Atmospheric Radiation Measurement (ARM) program it turned out that the scheme was able to reasonably represent the time-average condensate and cloud fraction profiles and the diurnal cycle of shallow convective clouds, but that the proposed parameterization for the subgrid variability could not represent the strong tempo$\mathrm{ral} / \mathrm{spatial}$ variability associated with individual deep convective events. This default of the scheme was related to the simple parameterization of the subgrid-scale variability with the aid of diagnostic first-order turbulence theory [see also Cuxart et al., 2000].

[6] Therefore this short contribution is intended to complete the study of CB by introducing a convective contribution to the subgrid variability based on the convective mass flux. Similar ideas have also been developed independently by [Lenderink and Siebesma, 2000] to represent the diurnal cycle of shallow convection over land. The parameterization is based on two-dimensional CRM simulations for a 1-week period of the Tropical Oceans Global Atmosphere (TOGA) Coupled Ocean-Atmosphere Response Experiment (COARE) and a 4-day period of the ARM 1997 experiment, instead of using three-dimensional large-domain CRM runs over only a 2-day period as in CB. The modified parameterization for the subgrid variability including a turbulent and a convective contribution is presented in section 2 . In section 3 the scheme is evaluated in a mesoscale model for daily forecasts during one month of the TROCCINOX experiment over southern Brazil. The cloud and precipitation forecasts are evaluated using available satellite observations in the thermal infrared band and satellite-derived rain rates. Particular focus is given on the impact of the cloud scheme on the representation of the diurnal cycle of convection over land. A summary in section 4 and an assessment of the limits of the current approach conclude the study.

\section{Cloud Parameterization}

\subsection{Original Version}

[7] Briefly, recall that in a statistical cloud parameterization both the cloud fraction $N$ and the total condensate mixing ratio $r_{l}=r_{c}+r_{i}$, with $r_{c}$ the cloud liquid water mixing ratio and $r_{i}$ the cloud ice mixing ratio, can be defined either analytically [e.g., Bougeault, 1981; Mellor,
1977; Sommeria and Deardorff, 1977] or empirically (CB) as a unique function of the normalized saturation deficit

$$
Q_{1}=\bar{a}\left(\bar{r}_{w}-r_{\mathrm{sat}}\left(\bar{T}_{l}\right)\right) / \sigma_{s},
$$

where $r_{w}=r_{v}+r_{c}+r_{i}$ is the total water mixing ratio (vapor + condensate) and $r_{\text {sat }}$ the saturation mixing ratio. Furthermore, the "liquid water temperature" $T_{l}$ is expressed as

$$
\begin{aligned}
T_{l} & =T-L_{v} / C_{p m} r_{c}-L_{s} / C_{p m} r_{i} \\
& =\left(h_{l}-\left(1+r_{w}\right) g z\right) / C_{p m},
\end{aligned}
$$

where $h_{l}$ is the liquid water static energy, $g$ is the gravity constant, $z$ is height, $L_{v}$ and $L_{s}$ correspond to the latent heat of vaporization and sublimation, respectively, and $C_{p m}=C_{p}+$ $r_{w} C_{p v}$ is the heat capacity of moist air.

[8] The subgrid variability $\sigma_{s}$ of the air mass is expressed in terms of the variance of total water and liquid water temperature as

$$
\sigma_{s}=\left(\bar{a}^{2} \overline{r_{w}^{\prime 2}}-2 \bar{a} \bar{b} \overline{r_{w}^{\prime} T_{l}^{\prime}}+\bar{b}^{2} \overline{T_{l}^{\prime 2}}\right)^{1 / 2},
$$

where $a$ and $b$ are thermodynamic functions arising from a linearization of the function for the water vapor saturation mixing ratio (see $\mathrm{CB})$

$$
\bar{a}=\left(1+L \frac{\partial r_{\mathrm{sat}}\left(T_{l}\right)}{\partial T} / C_{p m}\right)^{-1}, \quad \bar{b}=\bar{a} \frac{\partial r_{\mathrm{sat}}\left(\bar{T}_{l}\right)}{\partial T} .
$$

Here $L$ and $r_{\text {sat }}$ are the latent heat and water vapor saturation mixing ratio that inside a given glaciation interval $T_{0}>T>$ $T_{1}$ are linearly interpolated as a function of temperature between their respective values for liquid water and ice, i.e., $L=(1-\chi) L_{v}+\chi L_{s}, r_{\mathrm{sat}}=(1-\chi) r_{\mathrm{satw}}+\chi r_{\text {sati }}$, with $\chi=$ $\left(T_{0}-T\right) /\left(T_{0}-T_{1}\right), T_{0}=273.16 \mathrm{~K}$, and $T_{1}=253 \mathrm{~K} . r_{\text {satw }}$ and $r_{\text {sati }}$ are the saturation mixing ratios over water and ice, respectively. Finally, note that overbars have been used in (1), (3), and (4) to designate quantities that should be understood as ensemble means or grid-average values.

[9] The suggested parameterization for $\sigma_{s}$ using vertical gradients of the conserved quantities as derived from turbulence theory (stationary equations for the second-order turbulent moments, neglecting higher-order terms) was

$$
\begin{aligned}
\sigma_{s t u r b}= & c_{\sigma} l\left[\bar{a}^{2}\left(\frac{\partial \bar{r}_{w}}{\partial z}\right)^{2}-2 \bar{a} \bar{b} C_{p m}^{-1} \frac{\partial \bar{h}_{l}}{\partial z} \frac{\partial \bar{r}_{w}}{\partial z}\right. \\
& \left.+\bar{b}^{2} C_{p m}^{-2}\left(\frac{\partial \bar{h}_{l}}{\partial z}\right)^{2}\right]^{1 / 2}
\end{aligned}
$$

with a proportionality constant $c_{\sigma}=0.3$ and a turbulent length-scale $l$ set to a constant value of $600 \mathrm{~m}$ above the boundary layer. Below the $600 \mathrm{~m}$ level, which constitutes a typical height of the boundary layer over the oceans, $l$ decreases linearly to zero at the surface. Unfortunately, it turned out that this parameterization is able to approximately capture the average profile of $\sigma_{s}$, but not the strong spatial and time variation with large values associated to deep convective events. 
[10] For completeness, the analytical cloud relations as suggested by $\mathrm{CB}$ for the cloud fraction $N$ and the cloud condensate content $r_{l}$ normalized by $\sigma_{s}$ are also recalled

$$
\begin{aligned}
N & =\max \left\{0, \min \left[1,0.5+0.36 \arctan \left(1.55 Q_{1}\right)\right]\right\} \\
\frac{\bar{r}_{l}}{\sigma_{s}} & =e^{\left(1.2 Q_{1}-1\right)}, \quad Q_{1}<0, \\
\frac{\bar{r}_{l}}{\sigma_{s}} & =e^{-1}+0.66 Q_{1}+0.086 Q_{1}^{2}, \quad 0 \leq Q_{1} \leq 2, \\
\frac{\bar{r}_{l}}{\sigma_{s}} & =Q_{1}, \quad Q_{1}>2 .
\end{aligned}
$$

To fix some numbers, a value $Q_{1}=0$ corresponds to $50 \%$ cloud fraction, whereas values of $Q_{1}<-4$ and $Q_{1}>2$ typically correspond to clear sky and totally covered conditions, respectively.

[11] The coupling between the prognostic microphysics and the diagnostic statistical schemes is achieved by replacing the condensation/evaporation tendencies in the prognostic equations for $r_{c}, r_{i}, r_{v}$ and $T$ by the following tendencies:

$$
\begin{aligned}
\left.\frac{\partial \bar{r}_{c}}{\partial t}\right|_{\text {cond/evap }} & =\left[(1-\chi) \bar{r}_{l}-\bar{r}_{c}^{*}\right] / \Delta t \\
\left.\frac{\partial \bar{r}_{i}}{\partial t}\right|_{\text {cond/evap }} & =\left[\chi \bar{r}_{l}-\bar{r}_{i}^{*}\right] / \Delta t \\
\left.\frac{\partial \bar{r}_{v}}{\partial t}\right|_{\text {cond/evap }} & =-\left.\frac{\partial \bar{r}_{c}}{\partial t}\right|_{\text {cond/evap }}-\left.\frac{\partial \bar{r}_{i}}{\partial t}\right|_{\text {cond/evap }} \\
\left.\frac{\partial \bar{T}}{\partial t}\right|_{\text {cond/evap }} & =L_{v} /\left.C_{p m} \frac{\partial \bar{r}_{c}}{\partial t}\right|_{\text {cond/evap }}+L_{s} /\left.C_{p m} \frac{\partial \bar{r}_{i}}{\partial t}\right|_{\text {cond/evap }},
\end{aligned}
$$

where $\bar{r}_{l}$ is diagnosed from (7), $\chi$ is the fraction of liquid and solid condensate defined previously, $\Delta t$ is the model time step, and the asterisks denote values at the previous time step.

\subsection{CRM Study}

[12] The numerical experiments in CB using the Meso$\mathrm{NH}$ model [Lafore et al., 1998] are reconducted here. However, the experiments are now run with a two-dimensional (2-D) version of the model over a 6-day period for TOGA-COARE [Krueger et al., 1996] and a 4-day period for ARM [Xie et al., 2002]. The 2-D model domain consists of 128 horizontal grid points $(\Delta x=2 \mathrm{~km})$ and 47 vertical levels; the boundary conditions are cyclic. The time-dependent forcing of the CRM is given by observed large-scale tendencies for moisture and humidity and also surface fluxes for the ARM case, whereas for the horizontal wind components a simple relaxation toward the observed wind is applied.

[13] All CRM results displayed in the following represent ensemble (domain) average quantities. As an example is shown in Figures 1a and $1 \mathrm{~b}$ of a comparison of the time evolution of $\sigma_{s}$ for the ARM experiment as simulated by the CRM and obtained from the parameterization using (5). The time evolution of the cloud mass flux $\left(\mathrm{kg} \mathrm{s}^{-1} \mathrm{~m}^{-2}\right)$, defined as

$$
M=\bar{\rho} N_{u} w ; r_{c}+r_{i}>0 ; w>0,
$$

with $N_{u}$ the area fraction of cloudy updrafts, $w$ the updraft velocity, and $\rho$ the air density, is also depicted for comparison. It appears that the parameterized values do not reflect the strong time variation of the variances (especially in the ARM case) that are strongly related to variations in the cloud mass flux. Note, however, that in the CRM, significant values of $\sigma_{s}$ are only present after hour 20, corresponding to the first deep convective event, as the tropospheric dynamics in the CRM are only spun up with the occurrence of the first convective event.

[14] Furthermore, Figure 2 (see also Figure 4 of $\mathrm{CB}$ ) also shows the time mean and the fluctuations of the $\sigma_{s}$ profiles for both the TOGA and the ARM case. Note the discontinuity in the $\sigma_{s}$ and mass flux profiles in the TOGA case at the zero isotherm that marks a discontinuity in vertical stability. Figures $2 \mathrm{a}$ and $2 \mathrm{~b}$ summarize the previous results that the parameterization (5) is able to produce reasonable time-mean "background" profiles but cannot produce the strong (time) variability of $\sigma_{s}$ related to convective activity. As a consequence, with the turbulent formulation alone, mid and upper tropospheric cloud condensate and cloud fraction tend to be underestimated in convective situations. Nevertheless, the variation in $Q_{1}(1)$, and the variation in the cloud fraction and the cloud condensate, is partly reflected by a change in the saturation deficit $\bar{r}_{w}-\bar{r}_{s}$ that is explicitly resolved by the model. The cloud mass flux is able to provide the variability in the cloud layer (Figures $2 \mathrm{c}$ and 2d). These results are consistent with the CRM study of $X u$ and Krueger [1991], who found a good correlation between the convective mass flux and cloud condensate in convective situation. Therefore, in section 2.3 a convective contribution to $\sigma_{s}$ is derived and evaluated with the aid of CRM data.

\subsection{Convective Contribution to $\sigma_{s}$}

[15] Using the top hat approximation, the variance of a quantity $s$ can be expressed as [see, e.g., Lappen and Randall, 2001]

$$
\overline{s^{\prime 2}}=N_{u}\left(1-N_{u}\right)\left(s^{c}-s^{e}\right)^{2},
$$

where the superscripts $c$ and $e$ denote averages over cloudy and environmental regions, respectively. However, as noted by the authors and A. P. Siebesma (personal communication), the top hat approximation reasonably represents the convective fluxes but not the variances and is also sensitive to the decomposition chosen, i.e., cloud/environment or updraft/downdraft. Therefore, instead of (10) we seek a simple expression as a function of the convective mass flux (a quantity that is also readily available from mass flux parameterizations):

$$
\sigma_{\text {sconv }}={\overline{s^{2}}}^{1 / 2} \approx M \frac{\left(s^{c}-s^{e}\right)}{w^{*} \rho^{*}}
$$

where $M$ is the cloud mass flux, $w^{*}$ is a convective-scale velocity, and $\rho *$ is a tropospheric density scale. Equation (11) is further simplified to

$$
\sigma_{\text {sconv }} \approx M \frac{\left(s^{c}-s^{e}\right)}{w^{*} \rho^{*}} \approx \alpha M f\left(z / z^{*}\right),
$$

using a vertical scaling function $f$. It turns out that $\sigma_{s}$ is mainly determined (especially in the Tropics and in the upper 

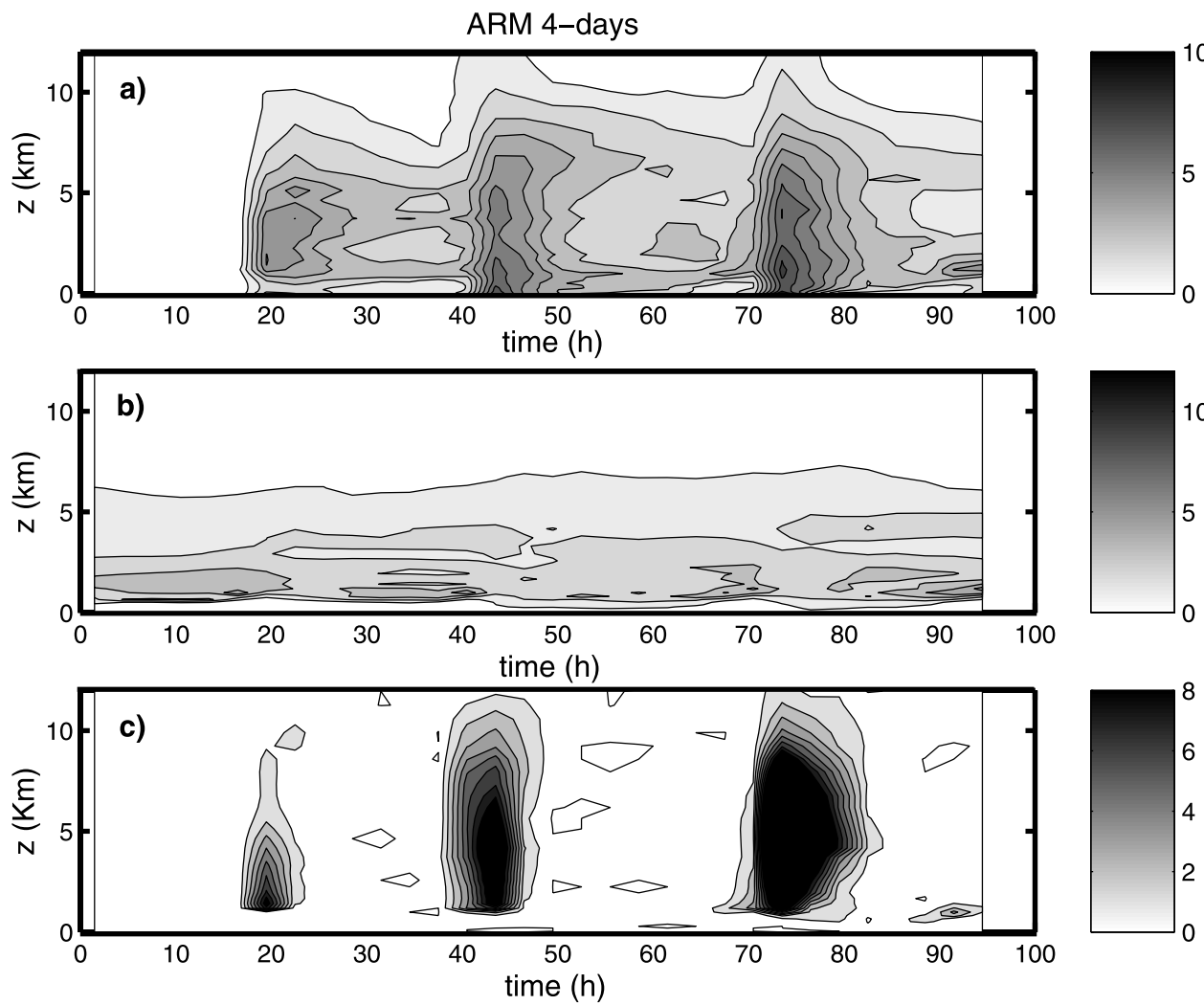

Figure 1. Time-height section of $\sigma_{s}$ for the ARM case (a) as simulated by the CRM and (b) parameterized using (5). (c) Evolution of the cloud mass flux. The values for $\sigma_{s}$ have been scaled by a factor of $1 \times 10^{4}$, and those of the mass fluxes have been scaled by a factor of $1 \times 10^{2}$.

troposphere) by the moisture variance [see also Liao and Rind, 1997; Tompkins, 2003]. The scaling function $f\left(z / z^{*}\right)$ can be determined from Figure 3, which displays CRM-derived cloud-environment differences for total water mixing ratio $r_{w}$ and $h_{l}$. For simplicity, the scaling function is set to $f=\bar{a}^{-1}$ which is proportional to the change of saturation mixing ratio with temperature (height). The function $f$ is also depicted in Figure 3, with typical values ranging between one and four.

[16] Finally, the value of the proportionality coefficient $\alpha$ can be obtained by minimizing the function $\left(\sigma_{s}^{2}-\sigma_{s t u r b}^{2}-\right.$ $\left.\sigma_{\text {sconv }}^{2}\right)^{2}$, where $\sigma_{s}^{2}$ are the CRM derived values for the TOGA and ARM periods (3-hourly mean values are used) and where $\sigma_{\text {sturb }}$ and $\sigma_{\text {sconv }}$ are replaced by the expressions (5) and (12), respectively. A value $\alpha=3 \times 10^{-3}$ is obtained using the cloud mass flux from the CRM. The order of magnitude of $\alpha$ can also be estimated from (11) using $s^{c}-$ $s^{e} \approx \bar{a}\left(r_{w}^{c}-r_{w}^{e}\right)=O\left(10^{-3}\right)$ (see Figure 3), an average tropospheric density $\rho^{*}=0.5 \mathrm{~kg} \mathrm{~m}^{-3}$ and a convective velocity-scale $w^{*}$ of $O(1) \mathrm{m} \mathrm{s}^{-1}$. However, in actual applications of (12) the cloud mass flux $M$ will be determined from a convection parameterization that might produce biased values of $M$ with respect to CRM data. Therefore, in individual applications the value of $\alpha$ might require an adjustment. Tests with different convection schemes suggest an uncertainty in the value of $\alpha$ in the range of a factor of two.

[17] The time evolution of $\sigma_{s}$ using the parameterization $\sigma_{s}^{2}=\sigma_{\text {sturb }}^{2}+\sigma_{\text {sconv }}^{2}$ with $\alpha=3 \times 10^{-3}$ is displayed in Figure $4 \mathrm{~b}$ and compared to the CRM-derived values. Furthermore, the time-averaged profiles over the entire periods and their variability are displayed in Figure 5. The results show that the introduction of a convective contribution clearly improves the representation of $\sigma_{s}$ concerning the time evolution and variability related to convective events. Using the turbulent contribution to $\sigma_{s}$ only, the parameterization reasonably reproduces the liquid water and cloud fraction for the moist TOGA-COARE case (consistent with the low variability in Figure $2 \mathrm{a}$ ), but only produces shallow convective clouds in the ARM case (not shown).

\section{Application During TROCCINOX \\ 3.1. Regional Simulations}

[18] In the following, the cloud parameterization is applied in a regional model, and its impact is evaluated on the basis of model simulations of tropical cloud systems and subsequent comparisons with satellite observations and rainfall retrievals. The model is the nonhydrostatic mesoscale model Meso-NH previously described. Its vertical resolution in the free tropospheric is set to $600 \mathrm{~m}$, and the horizontal resolution is $30 \mathrm{~km}$; the time step is $30 \mathrm{~s}$. Given the resolution, the model includes a parameterization for shallow and deep subgrid-scale convective transport and precipitation [Bechtold et al., 2001] based on the work of Kain and Fritsch [1993].

[19] During the period from 5 February to 7 March 2004 of the TROCCINOX experiment, a total of 30 daily 24-hour forecasts were run over a domain of $3000 \mathrm{~km} \times 3000 \mathrm{~km}$ covering São Paulo State in Brazil. The individual forecasts 

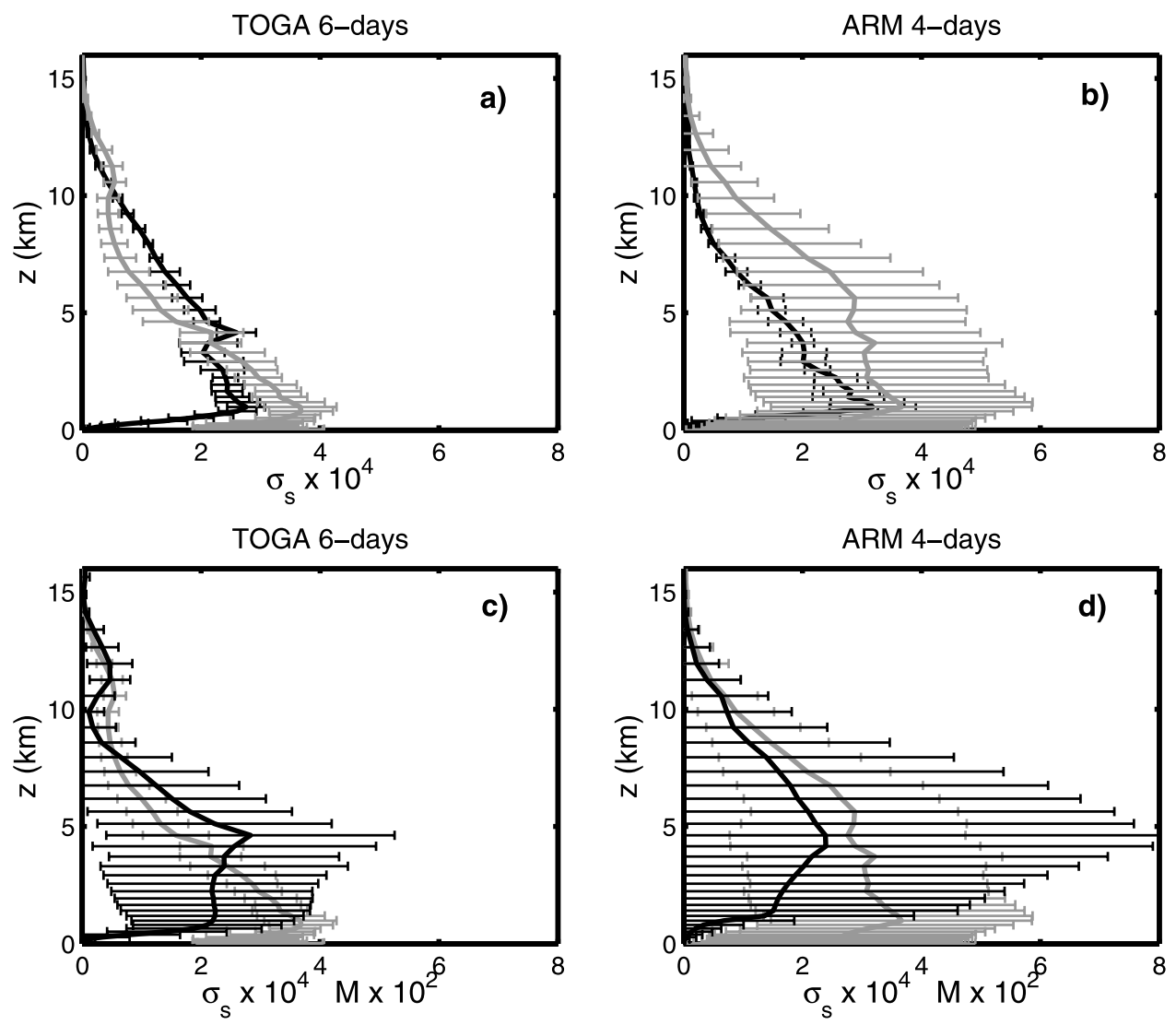

Figure 2. (a) and (b) Time-mean profiles of $\sigma_{s}$ (scaled by a factor $1 \times 10^{4}$ ) and their standard deviation for TOGA and ARM, as simulated by the CRM (gray shaded lines) and compared to parameterized values using (5). (c) and (d) The $\sigma_{s}$ profiles and their variance compared to the mean and variability of the convective mass fluxes (gray shaded profiles scaled by a factor $1 \times 10^{2}$ ) as computed from the CRM data.

were initialized with 12-hour ECMWF forecasts (first guess) based on the 1200 UTC ECMWF analysis. The fields included in the initial and boundary conditions of the numerical experiments are temperature, winds, and water vapor. No cloud initialization is performed, i.e., the mixing ratio of the liquid and ice water species build themselves during the course of the simulations. The control simulations (CTRL) use the cloud condensate from the prognostic all or nothing microphysical scheme only, while the simulations labeled SUBG additionally use the revised subgrid cloud parameterization.

\subsection{Model-to-Satellite Approach}

[20] The use of a model-to-satellite approach allows a direct comparison between simulated and observed cloud (radiation) fields [Chaboureau et al., 2000, 2002]. Synthetic brightness temperatures (BT) corresponding to the GOES-E infrared (IR) channel 4 in the thermal infrared window (10$11 \mu \mathrm{m}$ ) were computed, using the RTTOV version 7.1 code [Eyre, 1991; Saunders et al., 1999; Matricardi et al., 2001], which takes into account clouds as gray bodies [Chevallier and Bauer, 2003]. Maximum random cloud overlap is assumed. As an example, Figure 6 shows the results of the model-to-satellite approach at 0000 UTC 24 February 2004. The observed convection associated with the South Atlantic Convergence Zone (SACZ), is clearly seen with patchy BTs lower than $220 \mathrm{~K}$ along a line from Mato Grosso do Sul to the Atlantic Ocean (Figure 6c).

[21] In the resulting CTRL image, several convective cells appear at the right location (Figure 6a). However, the cloud patterns have a smaller spatial extent and significantly higher BTs than the observed ones. These discrepancies result in a domain averaged bias of $14 \mathrm{~K}$ and a correlation of 0.49 . The differences between the observation and the simulation are significantly reduced with the aid of the subgrid cloud parameterization (Figure 5b). The areas with BTs less than $240 \mathrm{~K}$ exhibit a spatial extent comparable to the observations, and the overall cloud pattern associated to the SACZ is fairly well reproduced. With the aid of the diagnostic cloud parameterization the domain averaged bias is reduced to $-4 \mathrm{~K}$ and the correlation increased to 0.75 . However, BTs less than $210 \mathrm{~K}$, corresponding to the most intense convective towers, are still absent in this mesoscale simulation.

[22] Furthermore, the subgrid cloud parameterization has a persistent positive impact on the simulations as shown by the 30-day time evolution of the BTs averaged over the whole domain (Figure 7). The BTs from the CTRL runs oscillate around $290 \mathrm{~K}$, where the oscillation is associated with the diurnal cycle of the convection. In contrast, the SUBG forecasts produce BTs with larger temporal variability, that follow more closely the time evolution of the observed GOES BTs. As a result, the correlation between 

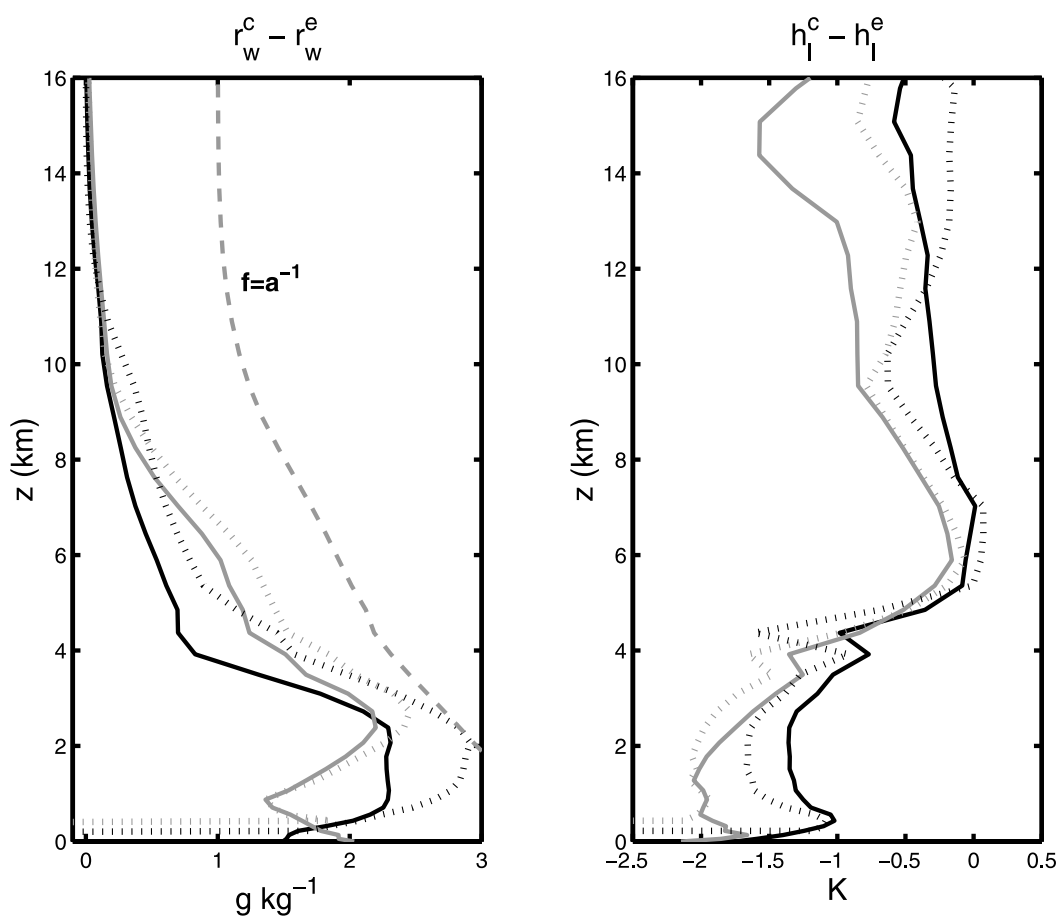

Figure 3. Mean cloud environment differences for TOGA (black lines) and ARM (gray shaded lines) computed from CRM for $r_{w}$ and $h_{l}$, where the cloud area is defined by $\overline{r_{c}}+\overline{r_{i}}>0$ (solid lines) and additionally by a vertical velocity threshold of $0.5 \mathrm{~m} \mathrm{~s}^{-1}$ (dotted lines). Additionally, the vertical scaling function $f=\bar{a}^{-1}$ is illustrated by the gray dashed line, typically bounded between values of one and four (note that $f$ has no unit).
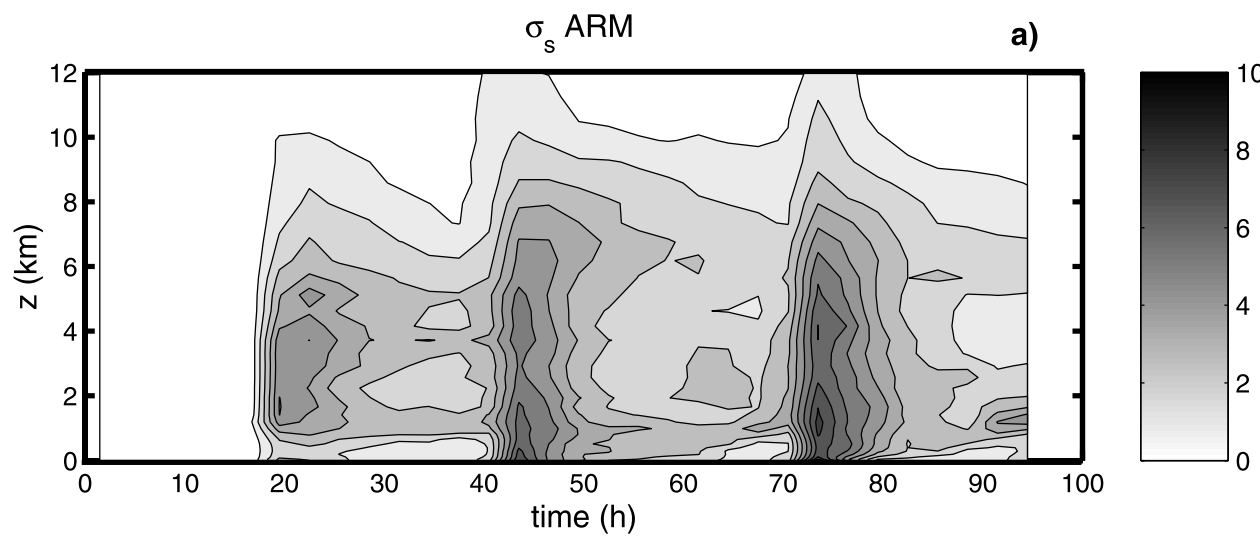

b)
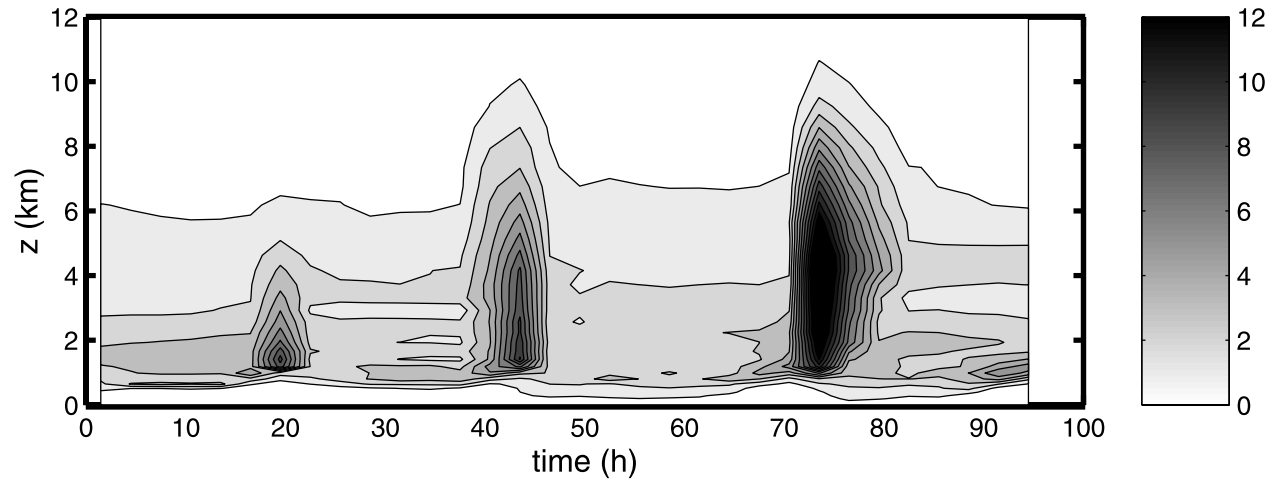

Figure 4. Time-height section of $\sigma_{s}$ for the ARM case (a) as simulated by the CRM and (b) with the new parameterization including the convective contribution (12); the values for $\sigma_{s}$ have been scaled by a factor of $1 \times 10^{4}$. 

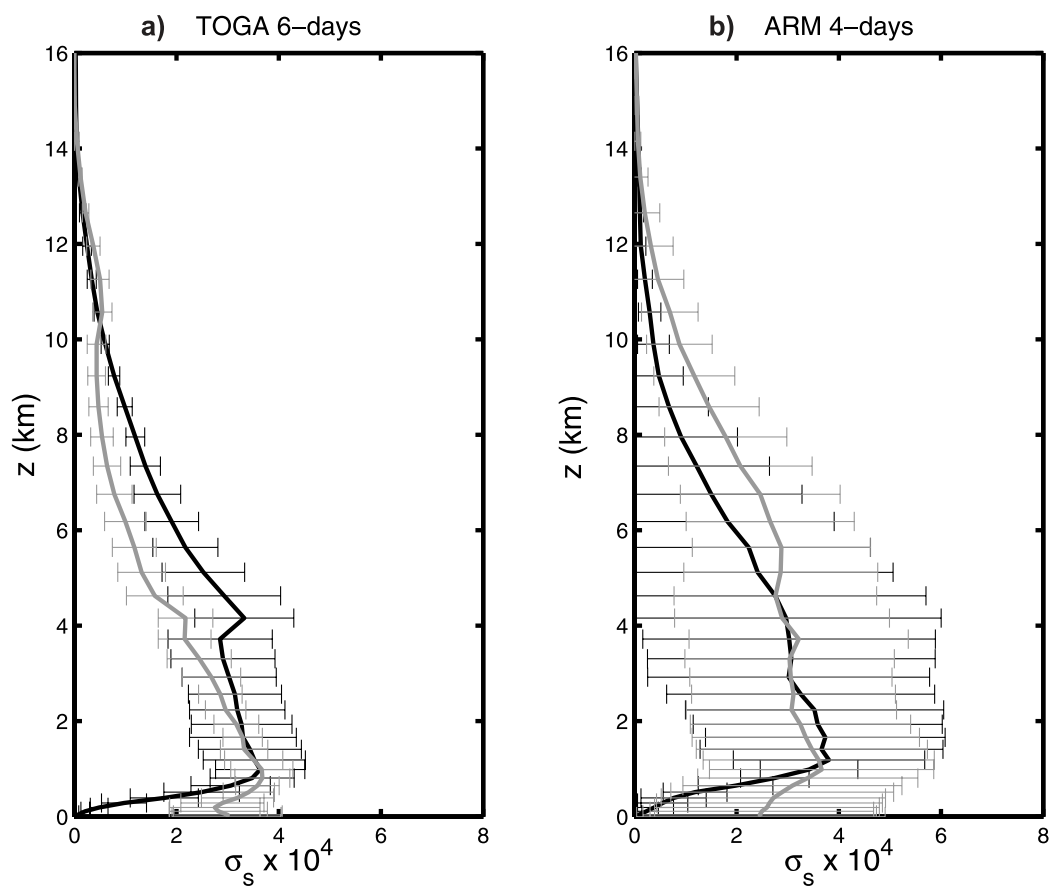

Figure 5. Time-mean profiles of $\sigma_{s}$ and their standard deviation for TOGA-COARE and ARM (a) as simulated by the CRM (gray shaded lines) and (b) compared to the parameterized values (black lines) using (5).

simulated and observed BTs increases from values between $0.2-0.6$ for the CTRL runs, to values of $0.4-0.8$ for the SUBG simulations (Figure $7 b$ ).

\subsection{Bauru Area}

[23] Next, a budget analysis similar to the one presented for the ECMWF model by Bechtold et al. [2004] is performed for the Bauru area $\left(20^{\circ} \mathrm{S}, 50^{\circ} \mathrm{W}\right)$ over a square of $150 \mathrm{~km} \times$ $150 \mathrm{~km}$. The composite diurnal cycle of the surface heat fluxes, IR BT, precipitation, convective available potential energy (CAPE), and convective inhibition (CIN), as obtained from the thirty 24-hour forecasts with model versions CTRL and SUBG, are displayed in Figure 8. The temporal resolution of both the forecasted and observed fields is 3 hours. The observed BTs from GOES, and the 3-hourly rain rates from the Tropical Rainfall Measurement Mission (TRMM) 3B42 product averaged over the Bauru area, are also plotted for comparison. The behavior of the diurnal cycle of CAPE, $\mathrm{CIN}$, and precipitation is remarkably similar to those previously obtained from both idealized midlatitude studies and studies for central Amazonia [Betts and Jakob, 2002; Bechtold et al., 2004]. The morning increase of CAPE, the drop in CIN and the onset of precipitation are all coupled to the diurnal cycle of the surface fluxes that have a maximum at 1200 local solar time (LST). The maximum observed precipitation rate (Figure 8d), however, occurs at 1800 LST, compared to $1500 \mathrm{LST}$ in the simulations. As reported by Yang and Slingo [2001], Guichard et al. [2004], and Tian et al. [2004], the too early occurrence of precipitation (convection) in the simulations compared to the observations is a common model error associated with the use of (diagnostic) convective parameterization schemes.
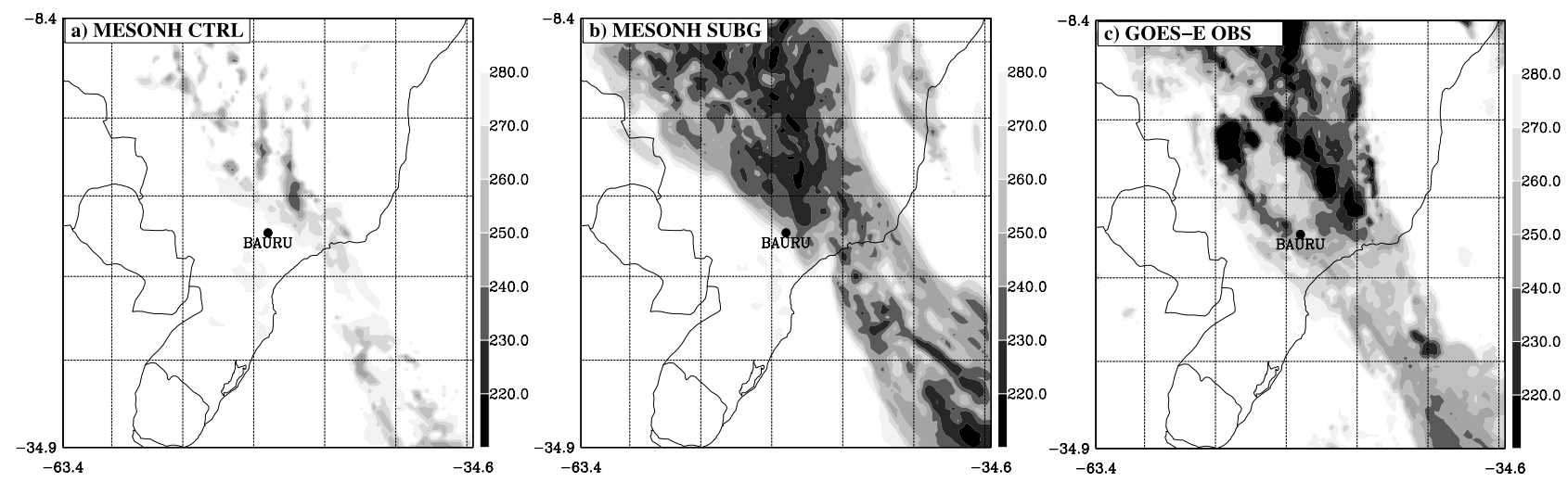

Figure 6. IR BTs (K) at 0000 UTC 24 February 2004 obtained from (a) the CTRL simulation, (b) the SUBG simulation, and (c) the GOES-E observation. 

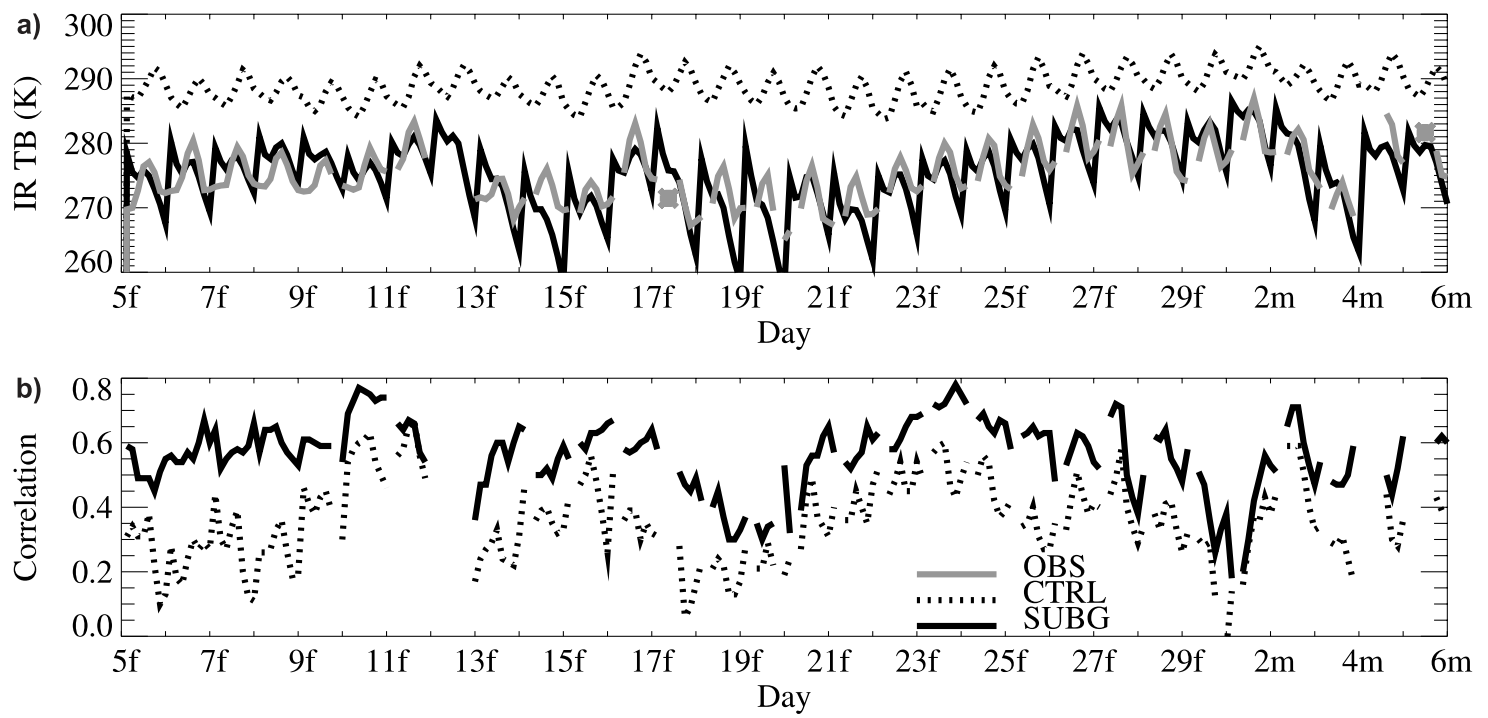

Figure 7. Time evolution of (a) the IR BTs (K) obtained from observation (gray line) from the CTRL simulations (dotted line), and from the SUBG simulations (solid line). (b) Correlation between simulated and observed IR BTs. On the time axis, f stands for February and $\mathrm{m}$ stands for March.
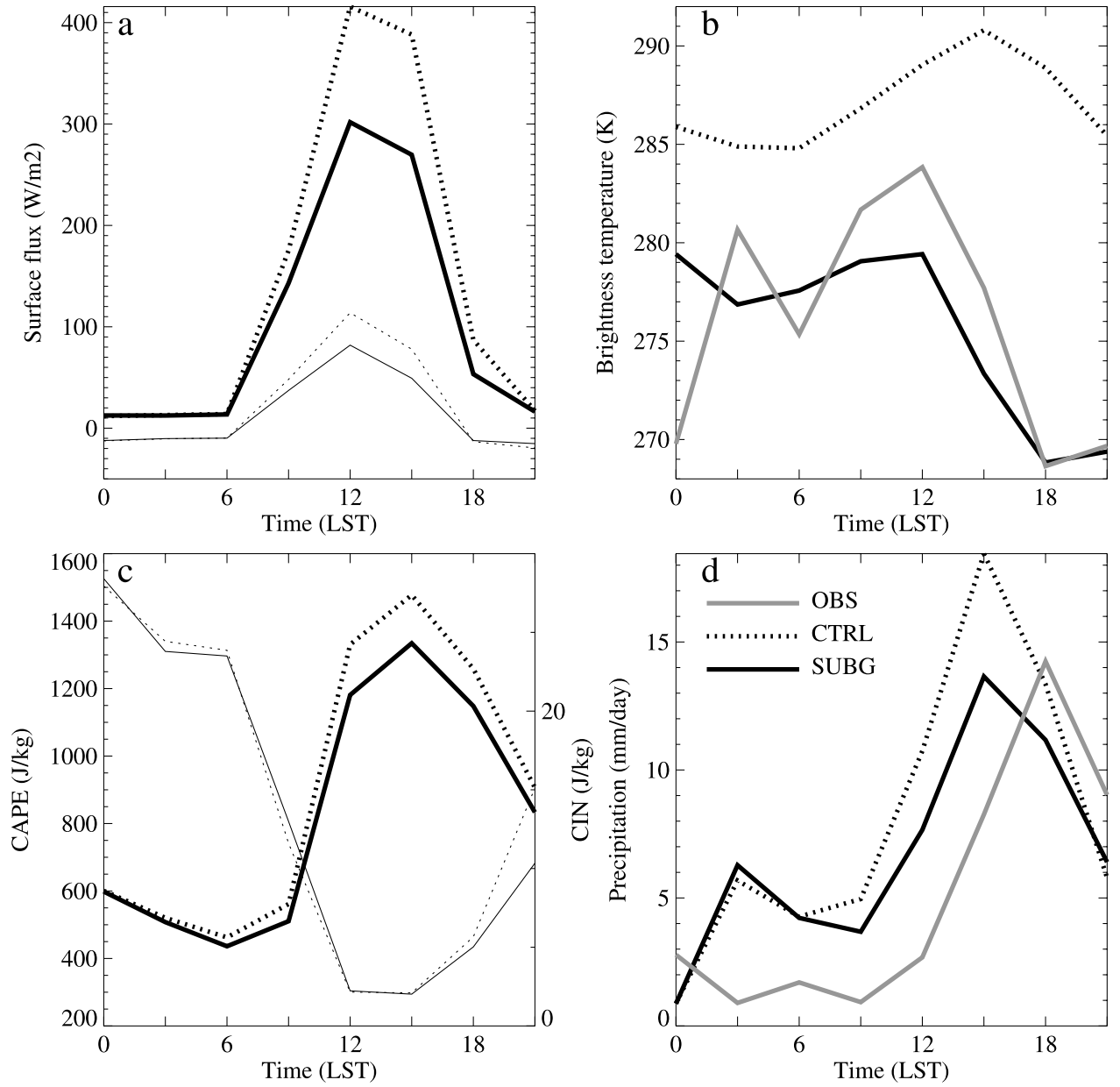

Figure 8. Diurnal cycle averaged over 30 days of (a) surface latent (thick lines) and sensible heat fluxes (thin lines), (b) observed and simulated infrared brightness temperature, (c) convective available potential energy CAPE (thick lines) and convective inhibition CIN (thin lines), and (d) observed and simulated precipitation rates. Results with model version CTRL are denoted by the dotted lines, and solid lines are used for the SUBG simulations. (b) and (d) GOES observations and the 3B42 retrievals denoted by the solid gray line, respectively. 

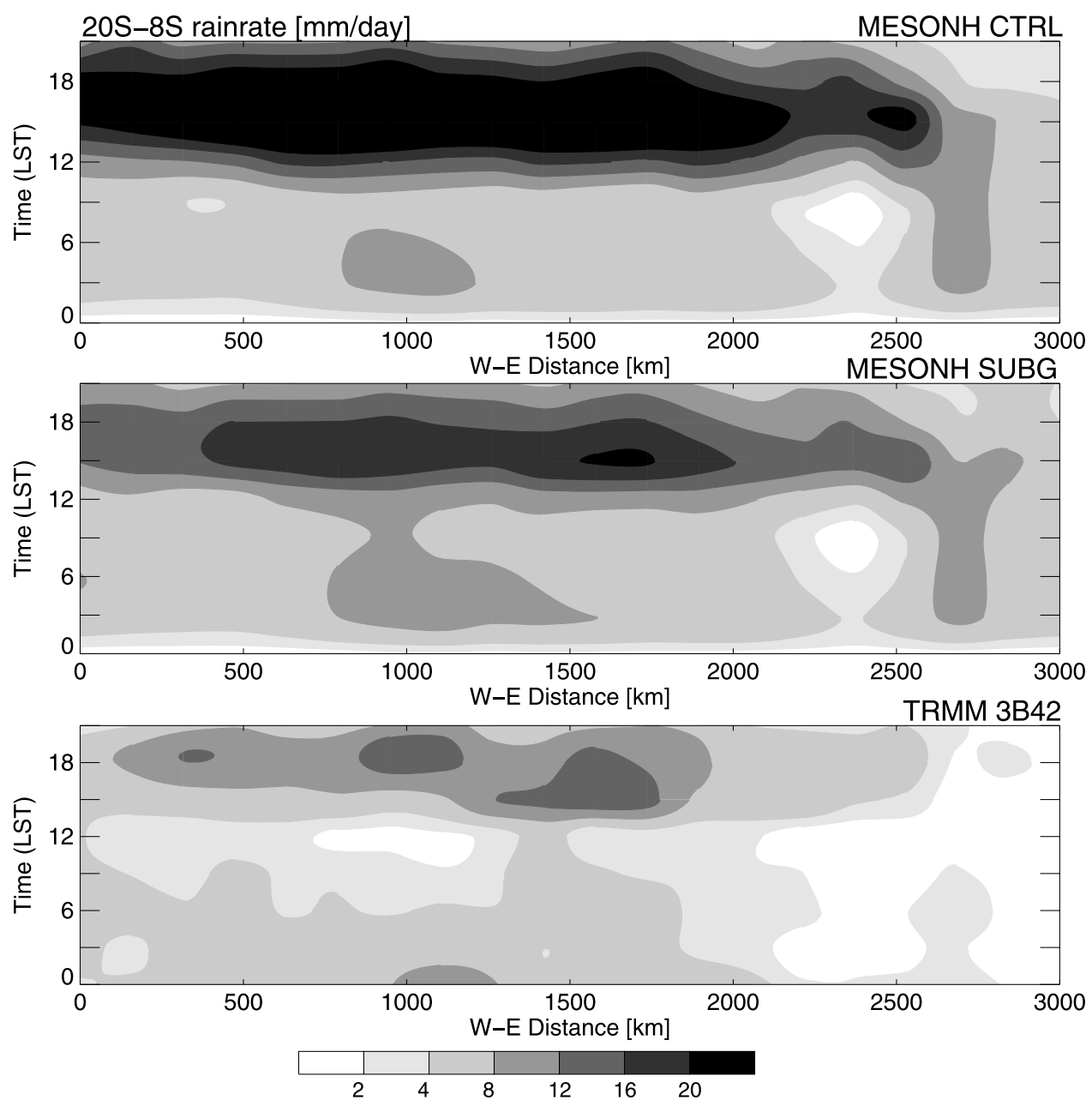

Figure 9. Hovmöller diagram of the diurnal cycle of precipitation $\left(\mathrm{mm} \mathrm{d}^{-1}\right)$ for the (a) CTRL and (b) SUBG simulations and (c) for TRMM observations. Fields are averaged between $20^{\circ} \mathrm{S}$ and $8^{\circ} \mathrm{S}$.

[24] Nevertheless, the SUBG experiments constitute a clear improvement over the CTRL experiments as the precipitation maximum is reduced, and in particular the diurnal cycle of BTs is now in phase with the observations (Figure $8 \mathrm{~b}$ ) with an amplitude of roughly $10 \mathrm{~K}$, whereas the CTRL experiments only produce a very weak diurnal cycle in BTs with an amplitude $<5 \mathrm{~K}$ (see also Figure 7a). The physical reasons for the strong impact of the subgrid cloud scheme on the diurnal cycle of convection reside (1) in a cloud/radiation effect that limits the surface heat fluxes (Figure 8a) and alters the tropospheric longwave radiative cooling, and (2) in a dynamical effect as the additional condensation (latent heat) produced by the subgrid cloud scheme alters the vertical stability of the troposphere. As discussed in the studies cited above, the impact of cloud/ radiation effects on the convective cycle is particularly strong in the Amazonas region, where the large-scale atmospheric forcing is weak.

\subsection{Regional Hovmöller Diagrams}

[25] Finally, with the aid of Hovmöller diagrams (longitude versus local time) the precipitation forecasts are further evaluated in the northern part of the domain, where precipitation is mostly produced by convection. Figure 9 compares the simulated precipitation rates to the TRMM-derived rain rates from the experimental product 3B42. Both, the model and observational data have been averaged over the latitude band of $20^{\circ} \mathrm{S}$ and $8^{\circ} \mathrm{S}$, the time resolution of the data is 3 hours.

[26] The Hovmöller plots show maximum precipitation rates over $10 \mathrm{~mm} \mathrm{~d}^{-1}$ that occur at 1500 LST for the simulations and at 1800 LST in the TRMM-based precipitation retrievals. With the use of the subgrid cloud parameterization, a significant decrease in the precipitation maxima is obtained. However, the SUBG rain rate is still to high compared to TRMM, and the model misses the distinct precipitation minimum around 0900 LST.

\section{Conclusions}

[27] A diagnostic statistical cloud parameterization is presented that provides both cloud condensate and fractional cloudiness from empirical CRM-derived functions. The scheme is coupled with a fully prognostic microphysical scheme via source/sink terms describing condensation/evaporation. The free parameter of the scheme is the subgrid variability $\sigma_{s}$ that contains contributions due to total water and temperature variance. Here it is proposed to parameterize $\sigma_{s}$ as a sum of a background "turbulent" contribution and a strongly variable "convective" contribution, that is proportional to the convective mass flux, a parameter that is 
readily available from mass flux convection parameterizations. CRM data for tropical and midlatitude convection has been used for a best fit of the diagnostic parameterization for $\sigma_{s}$. Therefore applying the current parameterization in an actual mesoscale model with a given convection parameterization might require some small retuning of the convective contribution, as a different convection parameterization might provide different mass fluxes. A portable version of the statistical cloud scheme written in FORTRAN 90 can be obtained from http://www.aero.obs-mip.fr/chajp/PUB/NCL/.

[28] The evaluation of the scheme has been performed in the context of routine regional cloud forecasts during the TROCCINOX campaign. Using GOES IR radiances and TRMM-derived rain rates, it is shown that with the aid of the statistical scheme the model significantly better reproduces the overall cloud field (BTs), but also better simulates the amplitude of the diurnal cycle of convection over land (both in precipitation, BTs and surface fluxes) compared to the control run with the all or nothing prognostic microphysical scheme. The observed strong impact of the cloud scheme on the simulation of the diurnal cycle is due to (1) the cloud/radiation interaction impacting on the surface fluxes and the tropospheric longwave radiative cooling rates and (2) on the change in atmospheric stability due to additional subgrid-scale condensation.

[29] However, the very simple current scheme has some evident shortcomings: (1) the diagnostic description of $\sigma_{s}$ that depends strongly on the convective mass flux might lead to an intermittent production of clouds if the mass fluxes produced by the convection parameterization are intermittent in time, and (2) the turbulent contribution of $\sigma_{s}$ that is important near inversions (e.g., boundary layer, melting level, tropopause) depends on a turbulent length scale for which a more accurate formulation or scaling [Khairoutdinov and Randall, 2002] might be sought depending on the different inversion heights present in the troposphere. An alternative to the current diagnostic formulation of $\sigma_{s}$ would be a prognostic statistical scheme as pioneered by Tompkins [2002] and currently pursued by A. M. Tompkins and S. A. Klein (personal communication, 2004). Such a scheme might lead to more realistic and smoother time/space distribution but its application might be more difficult if diagnostic applications are required as in specific assimilation schemes of satellite data.

[30] Acknowledgments. We wish to express our gratitude J. Duron for her help in the regional simulations. Our thanks go to two anonymous reviewers for their suggestions and to P. Marquet from Météo France and to J.-F. Mahfouf, M. Janisková, A. Tompkins, and M. Köhler from ECMWF for many stimulating discussions and applications. This research was supported by the TROCCINOX project funded by the European Commission under the contract EVK2-CT-2001-00122. Computer resources were allocated by IDRIS (projects 005, 569, and 1076). GOES-East data come from the NOAA Satellite Active Archive. We are grateful to the precipitation research team of Robert Adler at Laboratory for Atmospheres of the NASA Goddard Space Flight Center for posting the TRMM-based precipitation retrievals on the Web.

\section{References}

Arakawa, A., and W. H. Schubert (1974), Interaction of a cumulus cloud ensemble with the large-scale environment: Part I, J. Atmos. Sci., 31, $674-701$.

Bechtold, P., E. Bazile, F. Guichard, P. Mascart, and E. Richard (2001), A mass flux convection scheme for regional and global models, $Q$. J. R. Meteorol. Soc., 127, 869-886.
Bechtold, P., J.-P. Chaboureau, A. Beljaars, A. K. Betts, M. Köhler, M. Miller, and J.-L. Redelsperger (2004), The simulation of the diurnal cycle of convective precipitation over land in a global model, $Q . J . R$. Meteorol. Soc., 130, 3119-3137.

Betts, A. K., and C. Jakob (2002), Study of diurnal cycle of convective precipitation over Amazonia using a single column model, J. Geophys. Res., 107(D23), 4732, doi:10.1029/2002JD002264.

Bony, S., and K. Emanuel (2001), A parameterization of the cloudiness associated with cumulus convection; Evaluation using TOGA COARE data, J. Atmos. Sci., 58, 3158-3183.

Bougeault, P. (1981), Modeling the trade-wind cumulus boundary layer. Part I: Testing the ensemble cloud relations against numerical data, J. Atmos. Sci., 38, 2419-2439.

Chaboureau, J.-P., and P. Bechtold (2002a), A simple cloud parameterization derived from cloud resolving model data: Diagnostic and prognostic applications, J. Atmos. Sci., 59, 2362-2372.

Chaboureau, J.-P., J.-P. Cammas, P. Mascart, J.-P. Pinty, C. Claud, R. Roca, and J.-J. Morcrette (2000), Evaluation of a cloud system life-cycle simulated by Meso-NH during FASTEX using METEOSAT radiances and TOVS-3I cloud retrievals, Q. J. R. Meteorol. Soc., 126, 1735-1750.

Chaboureau, J.-P., J.-P. Cammas, P. Mascart, J.-P. Pinty, and J.-P. Lafore (2002b), Mesoscale model cloud scheme assessment using satellite observations, J. Geophys. Res., 107(D17), 4301, doi:10.1029/ 2001JD000714.

Chevallier, F., and P. Bauer (2003), Model rain and clouds over oceans: Comparison with SSM/I observations, Mon. Weather Rev., 131, 12401255 .

Cuijpers, J. W. M., and P. Bechtold (1995), A simple parameterization of cloud water related variables for use in boundary layer models, J. Atmos. Sci., 52, 2486-2490.

Cusack, S., J. M. Edwards, and R. Kershaw (1999), Estimating the subgrid variance of saturation, and its parameterization for use in a GCM cloud scheme, Q. J. R. Meteorol. Soc., 125, 3057-3076.

Cuxart, J., P. Bougeault, and J.-L. Redelsperger (2000), A turbulence scheme allowing for mesoscale and large-eddy simulations, $Q . J . R$. Meteorol. Soc., 126, 1-30.

Eyre, J. R. (1991), A fast radiative transfer model for satellite sounding systems, Tech. Rep. 176, Eur. Cent. for Medium-Range Weather Forecasts, Reading, U. K.

Guichard, F., et al. (2004), Modelling the diurnal cycle of deep precipitating convection over land with cloud-resolving models and single-column models, Q. J. R. Meteorol. Soc., 130, 3139-3172.

Kain, J. S., and J. M. Fritsch (1993), Convective parameterization for mesoscale models: The Kain-Fritsch scheme, Meteorol. Monogr., 46, $165-170$.

Khairoutdinov, M. F., and D. A. Randall (2002), Similarity of deep continental cumulus convection as revealed by a three-dimensional cloudresolving model, J. Atmos. Sci., 59, 2550-2566.

Krueger, S. K., D. Gregory, M. W. Moncrieff, J.-L. Redelsperger, and W.-K. Tao (1996), GCSS Working Group 4: First Cloud-Resolving Model intercomparison Project, case 2, technical report, Meteorol. Dep., Univ. of Utah, Salt Lake City.

Lafore, J.-P., et al. (1998), The Meso-NH atmospheric simulation system. Part I: Adiabatic formulation and control simulations. Scientific objectives and experimental design, Ann. Geophys., 16, 90-109.

Lappen, C.-L., and D. A. Randall (2001), Toward a unified parameterization of the boundary layer and moist convection. Part I: A new type of mass-flux model, J. Atmos. Sci., 58, 2021-2036.

Lenderink, G., and A. P. Siebesma (2000), Combining the massflux approach with a statistical cloud schemes, in Proceedings of 14th Symposium on Boundary Layers and Turbulence, Aspen, USA, pp. 66-69, Am. Meteorol. Soc., Boston, Mass.

Liao, X., and D. Rind (1997), Local upper tropospheric/lower stratospheric clear-sky water vapor and tropospheric deep convection, J. Geophys. Res, $102,19,543-19,557$.

Lohmann, U., N. McFarlane, L. Levkov, K. Abdalla, and F. Albers (1999), Comparing different cloud schemes of a single column model by using mesoscale forcing and nudging technique, J. Clim., 12, 438-461.

Matricardi, M., F. Chevallier, and S. Tjemkes (2001), An improved general fast radiative transfer model for the assimilation of radiance observations, Tech. Rep. 345, Eur. Cent. for Medium-Range Weather Forecasts, Reading, U.K.

Mellor, G. L. (1977), The Gaussian cloud model relations, J. Atmos. Sci., 34, 356-358.

Price, J. D., and R. Wood (2002), Comparison of probability density functions for total specific humidity and saturation deficit humidity, and consequences for cloud parametrization, Q. J. R. Meteorol. Soc., 128, 2059-2072

Ricard, J. L., and J. F. Royer (1993), A statistical cloud scheme for use in an AGCM, Ann. Geophys., 11, 1095-1115. 
Saunders, R., M. Matricardi, and P. Brunel (1999), An improved fast radiative-transfer model for assimilation of satellite radiance observations, Q. J. R. Meteorol. Soc., 125, 1407-1425.

Smith, R. (1990), A scheme for predicting layer clouds and their water content in a general circulation model, Q. J. R. Meteorol. Soc., 116, $435-460$.

Sommeria, G., and J. W. Deardorff (1977), Subgrid-scale condensation in models of nonprecipitating clouds, J. Atmos. Sci., 34, 344-355.

Teixeira, J. (2001), Cloud fraction and relative humidity in a prognostic cloud fraction scheme, Mon. Weather Rev., 129, 1750-1753.

Tian, B., B. J. Soden, and X. Wu (2004), Diurnal cycle of convection, clouds, and water vapor in the tropical upper troposphere: Satellites versus a general circulation model, J. Geophys. Res., 109, D10101, doi:10.1029/2003JD004117.

Tiedtke, M. (1989), A comprehensive mass flux scheme for cumulus parametrization in large-scale models, Mon. Weather Rev., 117, 17791800.

Tiedtke, M. (1993), Representation of clouds in large-scale models, Mon Weather Rev., 121, 3040-3061.

Tompkins, A. M. (2002), A prognostic parameterization for the subgridscale variability of water vapor and clouds in large-scale models and its use to diagnose cloud cover, J. Atmos. Sci., 59, 1917-1942.
Tompkins, A. M. (2003), Impact of temperature and humidity variability on cloud cover assessed using aircraft data, Q. J. R. Meteorol. Soc., 129, $2151-2170$

Wood, R., and P. R. Field (2000), Relationships between total water, condensed water, and cloud fraction in startiform clouds examined using aircraft data, J. Atmos. Sci., 57, 1888-1905.

Xie, S., et al. (2002), Intercomparison and evaluation of cumulus parameterizations under summertime midlatitude continental conditions, $Q$. J. R. Meteorol. Soc., 128, 1095-1135.

Xu, K.-M., and S. K. Krueger (1991), Evaluation of cloudiness parameterizations using a cumulus ensemble model, J. Atmos. Sci., 119, 342-367. Xu, K.-M., and D. A. Randall (1996), A semi-empirical cloudiness parameterization for use in climate models, J. Atmos. Sci., 53, 3084-3102.

Yang, G.-Y., and J. Slingo (2001), The diurnal cycle in the tropics, Mon. Weather Rev., 129, 784-801.

P. Bechtold, European Centre for Medium-Range Weather Forecasts, Reading RG29AX, UK. (peter.bechtold@ecmwf.int)

J.-P. Chaboureau, Laboratoire d'Aérologie, Observatoire Midi-Pyrénées, 14 avenue Belin, F-31400 Toulouse, France. (jean-pierre.chaboureau@ aero.obs-mip.fr) 